\title{
EL RETO DE LA EQUIDAD EDUCATIVA. METAS EDUCATIVAS 2021
}

\section{THE CHALLENGE OF EDUCATIONAL EQUITY. EDUCATIONAL GOALS 2021}

Juan Antonio Giménez Beut

\begin{abstract}
In relation to the goal of "Investing more and investing better" of the project of "Goals 2021: The education we want for the generation of the Bicentennials", we analyze the progress made in recent years and the challenges still pending for these next three years. There have been important advances in all the investment indicators of the Ibero-American education systems, although the academic results are not proportional to them, as we can see in the article. It is important to consider the great internal contrasts of each country and the linking of the results to the sociocultural and economic level of the families. Inclusion policies that favor equity will be necessary. The near economic indicators allow to continue the investment line although in a discreet way. It also happens with the HDI that is still far from reaching the desired levels. Faced with this situation, and apart from external investment, the analysis of the data collected leads us to conclude that we must invest in improving the management of the educational systems themselves. In this process, the role of teachers is fundamental. Other lines of action are proposed in order to finish promoting this line of growth.
\end{abstract}

Key words: investment, equity, education systems, financing.

\section{RESUMEN}

En relación a la meta de "Invertir más e invertir mejor" del proyecto de "Metas 2021: La educación que queremos para la generación de los Bicentenarios", se analizan los avances realizados en estos últimos años y los retos aún pendientes para estos tres próximos años. Se han producido importantes avances en todos los indicadores de inversión de los sistemas educativos Iberoamericanos, aunque los resultados académicos no sean proporcionales a los mismos como podremos comprobar en el artículo. Es importante tener en cuenta los grandes contrastes internos de cada país y la vinculación de los resultados al nivel sociocultural y económico de las familias. Serán necesarias políticas de inclusión que favorezcan la equidad. Los indicadores económicos próximos permiten continuar la línea de inversión aunque de manera discreta. Igualmente sucede con el IDH que aún dista de alcanzar los niveles deseados. Ante esta situación, y al margen de la inversión externa, el análisis de los datos recogidos nos lleva a concluir que se debe invertir en mejorar la gestión de los propios sistemas educativos. En este proceso es fundamental el papel de los docentes. Se proponen a su vez otras líneas de actuación para acabar de impulsar esta línea de crecimiento.

Palabras clave: inversión, equidad, sistemas educativos, financiación.

Fecha de recepción: 1 de enero de 2019

Fecha de aceptación: 29 de enero de 2019 


\section{INTRODUCCIÓN}

Tal vez no todas las personas sean conscientes de la importancia de la educación para la sociedad, la economía o el bienestar de sus miembros; pero no son ajenas a ellas las instituciones educativas nacionales e internacionales. Sin embargo, aun teniendo clara la importancia de este fin -que es la educación en sí misma- no hemos alcanzado un mecanismo idóneo para aprovechar el potencial de los sistemas educativos nacionales. Existen países igualados en el ranking internacional con sistemas educativos diametralmente opuestos. Así pues, aunque el suministro de recursos es necesario en estos sistemas educativos, no existe una relación directa entre esta inversión y el éxito de los mismos, pues existen múltiples variables que determinan sus resultados.

Los sistemas educativos han sido utilizados en el tiempo como mecanismos para promover la educación religiosa, para consolidar sistemas políticos, para impulsar divisiones o uniones territoriales, para formar a los trabajadores requeridos por la demanda laboral... Aunque sus fines puedan ser muchos -unos más lícitos que otros- es innegable su valor para las personas que componen las sociedades.

Es, de hecho, uno de los principales elementos vertebradores de la sociedad, facilitando en mayor o menor media la interrelación de los grupos e individuos, transmitiendo de una generación a otros sus saberes, promoviendo el bienestar de muchos de sus miembros al dotarlos de herramientas (no sólo instrumentales, también morales, afectivas o de interrelación...) que le permitan mejorar sus existencia y la del conjunto de las personas con las que convive.

Así pues, y habida cuenta de la relevancia de la educación en la vertebración y futuro de un país, es lógico que sea sustentada por el propio Estado. Si aterrizamos en nuestra realidad presente, son muchos los retos que requieren de la educación: la integración de la personas migrantes, la competitividad de sectores en riesgo, la posibilidad de mejorar las aspiraciones personales, la difusión de unos determinados valores, la innovación y la mejora constante de la propia humanidad etc. Y para los países en desarrollo es la herramienta con mayúsculas para dar ese "salto" esperado.

El éxito del sistema educativo no sólo depende de las buenas intenciones e inversiones del Estado. Es fundamental la percepción que los miembros de esta sociedad tengan de la utilidad de esta educación. Las personas con mayores niveles educativos obtienen mayores ingresos salariales; pero desgraciadamente hay, cada vez más, muchas excepciones. Aunque no existe una relación directa, es innegable que es positiva para los individuos y para los países el contar con una formación adecuada y suficiente. Nadie desea que sus descendientes tengan menos formación que ellos mismos, luego es valorada por los mismos.

Conscientes de esta importancia, y en el contexto de los países del área iberoamericana que están analizando aquí, se hace necesario partir de la XX Cumbre Iberoamericana de Jefas y Jefes de Estado y de Gobierno de 2010 aprobó el proyecto "Metas 2021: La educación que queremos para la generación de los Bicentenarios".

En el momento actual se está procediendo a revisar las metas planteadas para el año 2021 en materia de educación. Es el propósito del presente artículo revisar algunos aspectos vinculados con la meta número 10, a saber, "Invertir más e invertir mejor". 
Para esta meta se establecieron las siguientes metas específicas e indicadores:

Figura 1. Metas e Indicadores

\begin{tabular}{|c|c|c|}
\hline Meta específica 26 & Indicador 37 & Nivel de logro \\
\hline $\begin{array}{l}\text { Aumentar el esfuerzo económico } \\
\text { de cada país para el logro de las } \\
\text { Metas } 2021\end{array}$ & $\begin{array}{l}\text { Elaboración de un plan de } \\
\text { financiamiento por cada país en el } \\
\text { año } 2012 \text { para el logro de las Metas } \\
\text { y actualizarlo periódicamente }\end{array}$ & $\begin{array}{l}\text { Aprobar un plan en cada } \\
\text { país, evaluarlo y adaptarlo } \\
\text { cada tres años }\end{array}$ \\
\hline Meta específica 27 & Indicador 38 & Crear un Fondo \\
\hline $\begin{array}{l}\text { Incrementar la solidaridad } \\
\text { internacional con los países que } \\
\text { manifiestan mayores dificultades }\end{array}$ & $\begin{array}{l}\text { Crear un Fondo Internacional } \\
\text { Solidario para la Cohesión } \\
\text { Educativa en el año } 2010 \text { con un } \\
\text { plan de acción hasta } 2021\end{array}$ & $\begin{array}{l}\text { Internacional Solidario y } \\
\text { conseguir que aporte entre el } \\
20 \% \text { y el } 40 \% \text { de lo que } \\
\text { necesitan los países y las } \\
\text { regiones con mayor retraso } \\
\text { educativo para el } \\
\text { cumplimiento de las metas }\end{array}$ \\
\hline
\end{tabular}

Fuente: elaborado a partir de la información extraída en OEI, 2010

Estudiaremos el esfuerzo económico realizado por los países iberoamericanos en educación en estos últimos años y analizaremos las mejoras obtenidas a nivel global, la eficacia de éstas y los retos pendientes.

En el Plan de evaluación y seguimiento de 2016 (Miradas sobre Educación en Iberoamérica) se valoraron los avances en la educación infantil que se habían planteado como reto en informes anteriores. Ya se habían constatado los avances en la generalización de la educación primaria y un ligero despunte de la educación secundaria.

En el Plan de 2018 (p. 22-23) se muestra el trabajo del Instituto de Evaluación (IESME) de la OIE en cuanto a la vinculación de las metas 2021 con los Objetivos del Desarrollo Sostenible (ODS) de la Agenda de la Educación 2030. Allí se puede comprobar cómo la Meta 10 ("Invertir más y mejor") se relaciona con la 4a "Entornos de aprendizaje eficaces"; y no es baladí esta relación.

Aun cuando es posible registrar importantes avances en la región, conviene destacar también algunos retos que el informe Miradas sobre educación e Iberoamérica (2016) pone de manifiesto. Entre otros, la necesidad de ampliar la oferta de educación técnico-profesional para aquellos alumnos que terminan la educación secundaria; aumentar las tasas de matriculación en educación secundaria para alcanzar tasas de matriculación que den cobertura a la práctica totalidad de los alumnos; avanzar en un mejor conocimiento de la situación educativa de los colectivos más vulnerables (minorías étnicas, poblaciones originarias y afrodescendientes) mejorando la calidad educativa y superando las desigualdades.

\section{INVERSIÓN ECONÓMICA ACTUAL EN EDUCACIÓN}

En la Declaración de Incheon (UNESCO, 2015) se plantea en el documento 4 el "Garantizar una educación inclusiva y equitativa de calidad y promover oportunidades de aprendizaje permanente para todos". Y entre sus propósitos aparece el siguiente punto 14:

Estamos por tanto decididos a aumentar el gasto público en educación, de acuerdo con el contexto nacional, e instamos a que se cumplan los objetivos de referencia internacionales y regionales de 
asignar de forma eficiente a la educación al menos entre un 4\% y un $6 \%$ del producto interno bruto o al menos entre un 15\% y un $20 \%$ del total del gasto público (p. 99)

Según el Banco Mundial ${ }^{1}$, en el espacio de América Latina y El Caribe nos encontramos con una media entre $4,7 \%$ a $5 \%$ de inversión respecto del PIB del país. Pero, habría que comprobar si al final, este montante es suficiente para las necesidades del país, porque el porcentaje del PIB depende del potencial económico del propio país, y dos países con el mismo porcentaje pueden representar inversiones muy diferentes.

Cuando se analizan con detalle estas inversiones se puede observar que son absorbidas en gran parte por la partida de los salarios (Giorgi, J., 2017). Como el crecimiento de la economía en este entorno es muy ligero y las familias soportan una importante carga de gasto en esta partida, debería enfocarse la mirada hacia la eficacia del propio sistema más que al volumen de la inversión. Sobre todo, al analizar los resultados en las pruebas PISA, en donde, a pesar del esfuerzo presupuestario, no se han obtenido las mejorías esperadas.

La mayoría de los países han mejorado la escolarización de la educación primaria, por lo que tan sólo se espera un incremento del $2 \%$ en la inversión en esta etapa educativa. La educación infantil por su parte ya se extendió de manera generalizada durante estos últimos años. Queda pues pendiente la extensión de la educación secundaria que, aunque haya aumentado en torno al 15\% de la inversión, es baja, pues no se contabilizan los muchos alumnos que abandonan el sistema educativo (CEPAL, 2010).

Todos los indicadores educativos han mejorado: gasto total en educación, gasto por alumno inscripción escolar, capacitación de los maestros, perseverancia en la escuela, ratio profesor/alumno, alfabetización... (Banco Mundial, 2018).

\section{LA SITUACIÓN ECONÓMICA VERSUS NECESIDADES DE INVERSIÓN EN EDUCACIÓN}

Según los datos de 2015, es necesario un incremento anual del $0.12 \%$ del PIB para alcanzar los objetivos de las metas 2021 (excluyendo a México y Brasil).

Los costos de avanzar y cumplir con los compromisos educacionales (programas regulares, de alfabetización y educación básica para adultos, calidad e infraestructura educativa) asociados al proyecto Metas Educativas 2021, aumentan gradualmente. De esta forma, para el conjunto de Iberoamérica, el costo de iniciar con el compromiso en 2011 no supera los 8 mil millones de dólares, lo que representa solo el $0,18 \%$ del PIB regional que se proyecta para dicho año. En este año, el incremento de la inversión educativa es el más significativo, ya que los aumentos posteriores equivalen en promedio a poco más del $0,12 \%$ del PIB. (...)

Si se excluyen Brasil y México, que suman más del 40\% de la economía regional, los costos de avanzar hacia las metas en los países restantes se inician en alrededor de 4.500 millones de dólares $(0,17 \%$ del PIB). En 2015, el costo alcanzaría a poco más de 12.800 millones de dólares, y en 2021 alcanzaría los 43.600 millones de dólares ( 35 mil millones de euros). En promedio, el esfuerzo adicional requerido en cada año alcanzaría el 0,12\% del PIB. (OEI 2010, p. 168)

\footnotetext{
${ }^{1}$ https://datos.bancomundial.org/tema/educacion?locations=ZJ
} 
Si excluimos también a Portugal y España sería necesario un aumento adicional aproximado del $0.2 \%$. Como podemos ver en la tabla siguiente, se calcula una inversión necesaria del $1.46 \%$ para 2019 , del $1.62 \%$ para 2020 y del $0.21 \%$ para 2021 .

Tabla 1. Inversión necesaria para cumplir Metas 2021

\begin{tabular}{|c|c|c|c|c|}
\hline \multicolumn{3}{|c|}{ Iberoamérica } & \multicolumn{2}{c|}{$\begin{array}{c}\text { Iberoamérica sin México, Brasil, } \\
\text { Portugal y España }\end{array}$} \\
\hline Año & $\begin{array}{c}\text { Millones de } \\
\text { dólares }\end{array}$ & $\begin{array}{c}\text { Porcentaje } \\
\text { PIB }\end{array}$ & $\begin{array}{c}\text { Millones de dólares } \\
\text { Porcentaje PIB }\end{array}$ & 1.15 \\
\hline 2017 & 39668 & 0.73 & 16913 & 1.30 \\
\hline 2018 & 46941 & 0.84 & 19883 & 1.46 \\
\hline 2019 & 54737 & 0.95 & 23038 & 0.21 \\
\hline 2020 & 63121 & 1.06 & 26447 & 30035 \\
\hline 2021 & 72070 & 1.17 & 3062 & 1.62 \\
\hline
\end{tabular}

Fuente: Extracción de datos a partir de las aportaciones de CEPAL, sobre la base de los estudios de costos nacionales y del estudio preliminar de costos de CEPAL y OEI (CEPAL/OEI, 2010).

La pregunta es ahora la siguiente: ¿está la situación económica de estos países en condiciones de asumir estos costes?

A tenor de la perspectiva de crecimiento del PIB, se puede hablar de un crecimiento del 1.5\% (CEPAL, 2018, p.20), lo que supone una disminución de la previsión del mismo, pero con una gran disparidad según los países. En su conjunto podría ser asumida la inversión necesaria si se empleara casi toda la mejoría en educación.

Además de esta circunstancia, hay que tener en cuenta las condiciones particulares de cada uno de los países, pues muestran grandes diferencias entre ellos.

Esto lo podemos observar a través de diversos indicadores económicos. Si nos centramos en el endeudamiento, por ejemplo. Según los datos del CEPAL (2018, p.83), la deuda pública bruta en 2017 fue de 38.8\% PIB (1.4\% más que en 2016), aunque mejoró en el segundo semestre de 2018 (38\%). Igualmente, existen multitud de factores internos y externos que determinarán su evolución.

De los 18 países de la región de los que se dispone de información para el segundo trimestre de 2018, el Brasil sigue presentando el mayor nivel de endeudamiento (un 77,0\% del PIB), seguido por la Argentina (un 59,3\% del PIB) y Costa Rica (un 49,2\% del PIB). En el otro extremo se encuentra el Paraguay, con el menor nivel de endeudamiento público de la región (un 15,3\% del PIB), seguido del Perú (un 19,9\%) y de Guatemala (un 22,9\% del PIB) (p.83).

Los datos que se recogen del espacio iberoamericano nos sirven para ver una tendencia general pues, como estamos viendo, son muchas las diferencias entre los países. El verdadero problema común en gran parte ellos es la desigualdad entre los colectivos más y menos favorecidos y las 
consecuencias que esto tiene en el plano educativo. Analizar la inversión que realizan los países es importante, pero no es suficiente. Hay que conocer los entresijos de estas aportaciones de los Estados. Es necesario romper esta cadena de reproducción social, que es a su vez es la que perpetúa la pobreza. Precisamente es la educación la que posibilita romper esta inercia.

La pobreza y la desigualdad son los mecanismos principales que contribuyen a perpetuar la reproducción social y la limitación de la movilidad: bajos ingresos, condiciones desfavorables en el hogar, problemas de alimentación y salud, dificultades para mantener a los hijos en la escuela, bajo rendimiento escolar de los hijos, abandono temprano o escasa preparación, acceso a trabajos poco cualificados o con niveles de salarios inferiores y formación de una nueva familia que repite el esquema básico anterior (OIE, 2008, p. 70)

\section{LAS INVERSIONES MÁS URGENTES EN AMÉRICA LATINA}

El problema de muchos de los países del mundo -y que llama la atención en los del área iberoamericana- no es tanto el nivel educativo sino su asociación al nivel sociocultural y económico. Sobre todo al analizar los datos de los alumnos con mejores resultados (PISA²).

Pero no sólo es importante analizar este nivel, sino también el clima escolar. La forma de organizar las escuelas, el liderazgo, la comunicación con las familias o el compromiso del profesorado con los alumnos son algunos de los aspectos que pueden compensar los desequilibrios nombrados (OIE, 2008, P. 43).

Otra cuestión también importante es la redistribución de esta educación. La igualdad de oportunidades ha sido y es uno de los grandes retos de la humanidad -Naciones Unidas no ha dejado de insistir en ello-. ¿Qué se entiende por "igualdad de oportunidades"? En un primer acercamiento podemos identificar la expresión con la aportación de los recursos públicos en bien de todos sus miembros sin atender a su condición y eliminando las barreras de acceso a la educación. Sin embargo, esta aplicación de medios en su justa medida no es suficiente para analizar la justicia o "equidad“"(según la idea de Rawls) social. Distribuyendo por igual los recursos, no se consigue barrer estas diferencias de origen en las persona. Es evidente que precisarán más los que más necesitan. No obstante, no habría que olvidar a los que no precisan esa ayuda directa, pues también forman parte de la sociedad y tienen sus legítimas aspiraciones (función de bienestar/función de utilidad de Bermoulli-Nash).

Los hijos de familias con niveles sociocultural y económico altos tienen mayores expectativas de terminar sus estudios universitarios que el resto. Debería facilitarse que las personas que deseen y demuestren su voluntad y capacidad puedan alcanzar sus aspiraciones educativas independientemente de su origen social. Amartya Sen (2001) se refirió al término de "capacidades" para aludir a la obligación del Estado en facilitar que cada individuo sea capaz de desarrollar los proyectos que desee dentro de lo razonable. Este enfoque fue un punto de apoyo fundamental para el diseño del IDH (Índice de Desarrollo Humano) por la parte de la ONU para medir el nivel de desarrollo de un país.

En el último Informe sobre el Desarrollo Humano (ONU, 2016) encontramos una relación de países clasificados en cuatro bloques (desarrollo muy alto, alto, medio y bajo). En relación a los países del Caribe y América Latina, encontramos que la mayoría se encuentra en el nivel medio,

\footnotetext{
${ }^{2}$ https://webimages.iadb.org/publications/spanish/document/Am\%C3\%A9rica-Latina-y-el-Caribe-en-PISA-2015-\%C2\%BFCu\%C3\%A1ntostienen-bajo-desempe $\%$ C3\%B1o.pdf
} 
destacando en el IDH muy alto a Chile y Argentina y en el bajo a Haití. En cuanto a los datos globales, toda la región se eleva a un valor 0.751 .

Se muestran a continuación también otros indicadores:

Figura 2. IDH América Latina y Caribe

\begin{tabular}{lcccc}
\hline & $\begin{array}{c}\text { Índice de } \\
\text { Desarrollo } \\
\text { Humano }\end{array}$ & $\begin{array}{c}\text { IDH ajustado por } \\
\text { la Desigualdad } \\
\text { (pérdida total) }\end{array}$ & $\begin{array}{c}\text { Índice de } \\
\text { Desarrollo de } \\
\text { Género }\end{array}$ & $\begin{array}{c}\text { Índice de } \\
\text { Desigualdad de } \\
\text { Género }\end{array}$ \\
\hline $\begin{array}{l}\text { América Latina } \\
\text { y Caribe }\end{array}$ & 0.751 & $23.4 \%$ & 0.981 & 0.390 \\
\hline
\end{tabular}

Fuente: datos extraídos de (PNUD, 2016, p.29)

En este índice se combinan los aspectos que "una persona valora ser o hacer", las capacidades para hacerlas y las posibilidades de lograrlo. Esta sería otra forma de analizar la situación actual de este espacio. Pues bien, los principales obstáculos que según este informe dificultan el universalismo son:

- La intolerancia y la exclusión

- El poco poder de negociación

- La captura de la instituciones por las élites

- La reducción de la identidad propia

Habría que acometer políticas en estas cuatro líneas para asegurar la mejora social. El sistema educativo y estos cuatro elementos se retroalimentan mutuamente ayudándose entre sí. Y "para que el desarrollo humano llegue a todos, el crecimiento debe ser inclusivo" (ONU, 2016, p.11) En 1966 se publicó el "Equality of Educational Opportunity (Coleman, J. y Campbell, E. ${ }^{3}$ ). Un impresionante informe de 600.000 alumnos y 60.000 profesores. Lo llamativo de aquel informe es que las desigualdades no se eliminaban con la mayor inversión de dinero ni de medios. Eran más importantes los profesores y su pertenencia a la minoría africana (Balart, 2016). Así pues, tenemos que analizar bien qué es lo que hay que cambiar en cada uno de los países al margen de los factores materiales y la inversión (que también es necesaria). Y dar con las claves para entender qué está impidiendo superar estas desigualdades sociales. Habrá que valorar la rentabilidad formativa de la inversión realizada y reflexionar en torno a dos cuestiones: si la inversión es suficiente y si se está optimizando adecuadamente.

Según los datos de la OCDE (CEPAL, 2018), el gasto en educación primaria es del 6\% del PIB (muy por encima de los datos referidos a la educación secundaria). Pero los modelos para financiarlo son muy distintos. En países como Estados Unidos o Chile, por ejemplo, se ha optado por créditos subvencionados o garantizados por el Estado. En estos países es el propio estudiante quien al acabar sus estudios paga el coste de su formación (Balart, 2016). En otros, es el Estado quien organiza y financia el grueso de la educación del sistema. En otros, se complementa una red de centros públicos con otra de centros privados concertados (subvencionados). En cualquier caso, lo que hay que analizar es en qué medida los diferentes sistemas logran mejorar los niveles educativos del mayor número de personas.

\footnotetext{
3 http://blogbibliotecas.mecd.gob.es/2016/10/31/informe-coleman-50-aniversario/
} 
Así pues, encontramos entre los países de mayor desigualdad educativa a Perú y Chile. Se trata de países donde los rendimientos educativos están polarizados alrededor del nivel social y económico de los padres. La financiación de este tipo de países es una de los objetivos del Desarrollo del Milenio de Naciones Unidas ${ }^{4}$ (coor. Sachs, J.). Esther Duflo (2012) analiza la importancia de forzar a la escolarización de todos los alumnos, de reducir el absentismo de toda la población infantil.

La UNESCO (2008) también valora las mejoras experimentadas respecto a la escolarización universal, e igualmente hace ver las grandes desigualdades entre las capas sociales y la necesidad de mejorar la calidad de esta enseñanza.

Pese a las mejoras en la enseñanza preescolar y el elevado grado de cobertura de ésta, los niños más desfavorecidos no suelen beneficiarse en general de los programas de atención y educación de la primera infancia (AEPI) (p.1)

En el conjunto Iberoamericano estos serían (OEI, 2008) los tres grandes bloques de países en función de las tasas de escolarización y las desigualdades en el país:

Figura 3. Tasas de escolarización y desigualdades en los Estados Iberoamericanos

\begin{tabular}{|c|c|c|}
\hline & $\begin{array}{l}\text { Tasas de } \\
\text { Escolarización }\end{array}$ & $\begin{array}{l}\text { Desigualdades } \\
\text { internas }\end{array}$ \\
\hline $\begin{array}{l}\text { Cuba, Argentina, Uruguay, México, Chile y } \\
\text { Ecuador }\end{array}$ & Alta & Baja \\
\hline $\begin{array}{l}\text { Colombia, Panamá, Brasil, Costa Rica, Perú y } \\
\text { Venezuela }\end{array}$ & Media & Media \\
\hline $\begin{array}{l}\text { Nicaragua, El Salvador, República } \\
\text { Dominicana, Honduras y Guatemala }\end{array}$ & Baja & Alta \\
\hline
\end{tabular}

Fuente: elaborado a partir de la información en (OEI, 2008, p. 28)

Como se puede apreciar existe una correlación inversa entre las tasas de escolarización y las desigualdades internas. Y es que la propia extensión de la educación a la población escolar favorecerá un acceso al mercado laboral en mejores condiciones de igualdad rompiendo las barreras invisibles de las personas según el origen familiar.

\section{CARENCIAS ACADÉMICAS Y ENFOQUE DE LAS FUTURAS INVERSIONES}

Se hace ahora necesario valorar los resultados académicos en sí, y no sólo los datos económicos globales. Para ello vamos a tomar como referencia los resultados de pruebas estandarizas que, pese a sus limitaciones, puede presentarnos un panorama global de la situación.

En 1997, la OCDE y la UNESCO, en colaboración de algunos países (entre los que estaban Argentina, Brasil y Chile) y el Banco Mundial, crearon un programa mundial de indicadores educativos: el World Education Indciators Programme (WEI) que dio pie a la elaboración de los conocidos informes PISA. Era la forma de comparar los resultados educativos de diferentes países con diferentes sistemas, inversiones y modelos financiación.

Según los resultados obtenidos en 2015 en este informe PISA (Bos, M.S., Elías, A., Vegas, E. y Zoido, P., 2015) para la toda la región de América Latina y el Caribe se alcanza un bajo desempeño en todos los ámbitos.

${ }^{4}$ http://www.undp.org/content/undp/es/home/sustainable-development-goals.html 
Por ejemplo en ciencia no alcanzan un nivel básico el 50\% de los estudiantes, el 46\% en lectura o el 63\% en matemáticas. Es cierto que con contrastes entre el mejor en los tres aspectos que es Chile (35\%, 28\% y 63\% respectivamente) y el peor calificado que recae en la República Dominicana $(86 \%, 72 \%$ y $91 \%$ respectivamente). Pero en todo caso muy distantes de la media de la OCDE (21\%, 20\% y $23 \%$ respectivamente).

Si bien hemos visto que en líneas generales se ha aumentado la inversión y que existen posibilidades económicas de seguir haciéndolo, habría que articular mecanismos para hacer más efectiva la misma. Como se ha podido comprobar queda aún un largo recorrido para alcanzar los verdaderos objetivos para el 2021.

Hay que tomar decisiones a la hora de analizar la optimización de la inversión educativa realizada en estos países. Hay sistemas educativos más exitosos que otros ¿Cuáles son las claves de su éxito? ¿Qué es conveniente, invertir en todas las etapas educativas o crear un sistema restrictivo reduciendo la masa estudiante seleccionando poco a poco a los mejores? ¿Qué peso tiene la inversión en la formación inicial y permanente de los docentes? y otras tantas preguntas. Creemos que existen algunas líneas de actuación que podrían ayudar a mejorar los resultados y el éxito de los sistemas educativos.

La primera de ellas tiene que ver con el profesorado. El informe Coleman, A. Hanushek (2007) destacó la importancia del "valor añadido de los profesores". Es importante estimular e incentivar a los profesores. Henry Levin (2017), que hay mitos que no se sostiene, como son la importancia atribuida a la experiencia o los programas informáticos. Él demostraba que era más importante la capacidad para comunicarse oralmente o la ayuda entre iguales.

En gran parte de los países iberoamericanos se adolece de una débil formación del profesorado. Los profesores además, tienen una ardua tarea que deben realizar con pocos medios materiales y pedagógicos y una débil remuneración, lo que hace poco atractiva la profesión. Esta es precisamente una línea de trabajo, estimular la profesión docente.

La segunda línea de actuación que debería priorizarse podría ser la incorporación de orientadores escolares en los centros educativos. Como James Heckman (2004) ha estudiado, son muy importantes las habilidades no cognitivas en los alumnos para facilitar su éxito educativo. Habilidades como la responsabilidad, la apertura a nuevas experiencias, la extroversión, la amabilidad y la estabilidad emocional están estrechamente relacionadas con las habilidades cognitivas. Para poder desarrollar estas habilidades, y teniendo en cuenta la importancia del acompañamiento personal de los alumnos, reivindicamos el papel del orientador educativo como órgano que, junto a los profesores y tutores de los alumnos, actúen directamente con el alumnado, coordinen y aglutinen a los profesores en torno al desarrollo de estas habilidades, y creen vínculos con las familias implicándolas en el proceso formativo.

En tercer lugar hay que tener en cuenta las posibilidades de la formación en la web. Existen iniciativas que han demostrado su utilidad como son las MOOC (Massive Open Online Courses) o la Academia Khan (creada por Salman Khan) que lanzan cursos gratuitos de un altísimo nivel por las redes (Balart, 2016). Esto puede vencer las limitaciones presupuestarias y los desequilibrios entre grupos de personas en las sociedades modernas; aunque, eso sí, exige de un acceso -y un acceso libre- a la red para poder engancharse a un sinfín de posibilidades. 
En cuarto lugar el buen aprovechamiento de los recursos ya existentes. La cuestión de los recursos está más del lado de la calidad y la optimización de los mismos que de una cuestión linealmente de cantidad de inversión. En esta línea sería conveniente acometer una descentralización de las competencias en educación, una coordinación entre instancias, una asignación correcta de los recursos, la supervisión escolar... (Marcehsi, 2008):

El logro de las metas educativas exige incluir los factores determinantes de la calidad de la enseñanza una gestión eficiente de los recursos públicos. Ello supone una coherente descentralización de las competencias educativas entre los niveles central, regional municipal y escolar, la coordinación entre todos ellos, la agilidad en la adopción de decisiones y en la asignación de los recursos públicos, y una visión equilibrada de las repercusiones en el aprendizaje y en el rendimiento de los alumnos de un conjunto de decisiones sobre el funcionamiento de las escuelas: horario lectivo y horario escolar, calendario escolar y distribución de las vacaciones, costos del funcionamiento y del equipamiento escolar, plantilla de profesores, organización de la escuela, autoridad del equipo directivo y funciones de los supervisores escolares y de los responsables dela ejecución de la política educativa establecida. (p. 138)

Y en quinto lugar el incorporar nuevos objetivos educativos y la actualización de los métodos.

Según la UNESCO (2015, p. 138 y ss.) deberían incorporarse en los currículos nuevas competencias como las relacionados con la educación para la ciudadanía, la convivencia, las TIC o el desarrollo sostenible.

5.2. Temas emergentes relevantes para la educación futura en América Latina y el Caribe.

A continuación se discuten con mayor detalle algunos de los temas emergentes identificados como relevantes para la región, no contenidos explícitamente en los objetivos EPT-2015, en particular, la educación para la ciudadanía, la relación entre educación y las ciudades, el asunto de la convivencia escolar, el uso de las tecnologías de la información y comunicación para el aprendizaje, la educación para el desarrollo sostenible y el cambio climático, y la educación intercultural bilingüe. Asimismo, se sintetiza la visión de organizaciones no gubernamentales sobre los desafíos regionales para la educación post-2015.

Incidiendo en Informe Sobre Desarrollo Humano, se plantean aspectos relevantes que afectan a la metodología docente y que son necesarios en el siglo XXI (2016, p.13). Estos aspectos también marcarían otra línea de actuación que podrían servir de referencia para iniciar las reformas pendientes. Principalmente apostar por metodologías que desarrollen el pensamiento, las formas de pensar ("creatividad, pensamiento crítico, solución de problemas, toma de decisiones, aprendizaje"). También estimular las herramientas TIC y las competencias para usarlas. En tercer lugar aprender a trabajar con los otros y a comunicarse con los demás, lo que se puede lograr con la incorporación de metodologías cooperativas.

\section{CONCLUSIONES Y PROPUESTAS FINALES}

En relación a la meta de "Invertir más e invertir mejor" del proyecto de "Metas 2021: La educación que queremos para la generación de los Bicentenarios", se ha podido comprobar que ha se ha aumentado la inversión en el área iberoamericana, especialmente en la educación Infantil y Primaria. Los indicadores globales de inversión respecto al PIB o al gasto público nos dan una idea del peso que tiene la educación como prioridad, de los esfuerzos realizados y de la necesidad de seguir aumentando los mismos. 
En líneas generales, una mayor inversión en educación ha supuesto una mejoría en todos los indicadores que analizan los sistemas educativos: alfabetización, escolarización, reducción del abandono etc.

Esto ha reportado beneficios directos sobre la cohesión y justicia social, la mayor cualificación del empleo y una esperanza de los individuos respecto a su futuro. Sin embargo, los resultados académicos (PISA) de los mismos no son proporcionales al esfuerzo inversor.

El indicador global de la inversión de un país es un dato que, analizado de forma aislada, puede inducir a error. Son flagrantes las grandes disparidades encontradas en la región Iberoamericana respecto a la relación de la eficacia educativa y el nivel sociocultural y económico de procedencia de las familias. Es necesario invertir en políticas que corrijan esta falta de equidad entre los ciudadanos y los grupos sociales.

El crecimiento económico previsto para la región en líneas generales y el análisis de la tendencia hasta ahora obtenida nos hacen pensar que gran parte de los países del área en estudio podría alcanzar las metas 2021 propuestas si se priorizara la inversión de estos incrementos previstos para la educación.

Junto a esta premisa habría que adoptar medidas encaminadas a la optimización de los recursos. Es urgente dirigir la mirada hacia la buena gestión de los mismos y la eficacia de todos los estamentos del sistema. Al respecto se debería desarrollar un proceso de descentralización de la gestión y evaluación de la educación regulada.

En cuanto al uso de las nuevas cantidades presupuestarias debería priorizarse las dirigidas a los educadores: selección de los mejores docenes, incorporación de orientadores escolares y la formación en metodologías activas. En cuanto a la dotación material la inversión en recursos TIC para los centros escolares. 


\section{BIBLIOGRAFÍA}

Balart, P. (2016). Invertir en educación en un mundo globalizado. Madrid: RBA

Bos, M.S., Elías, A., Vegas, E. y Zoido, P. (2015). Pisa. América Latina y Caribe. BID \& CIMA. Recuperado de: https://publications.iadb.org/publications/spanish/document/Am\%C3\%A9rica-Latina-yel-Caribe-en-PISA-2015-\%C2\%BFCu\%C3\%A1ntos-tienen-bajo-desempe \%C3\%B1o.pdf

Comisión Económica para América Latina y el Caribe (CEPAL). (2010). Sobre la base de los estudios de costos nacionales y del estudio preliminar de costos de CEPAL y OEI. Comisión Económica para América Latina y el Caribe (CEPAL). Santiago de Chile: CEPAL. Recuperado de: https://repositorio.cepal.org/bitstream/handle/11362/3773/lcw327.pdf?sequence=1

Comisión Económica para América Latina y el Caribe (CEPAL). (2011). Desafíos para una educación con equidad en América Latina y el Caribe. Encuentro Preparatorio Regional 2011. Comisión Económica para América Latina y el Caribe (CEPAL). Buenos Aires: CEPAL. Recuperado de:

http://www.unesco.org/new/fileadmin/MULTIMEDIA/FIELD/Santiago/pdf/desafiosBuenos-Aires.pdf

Comisión Económica para América Latina y el Caribe (CEPAL). (2017). Estudio Económico de América Latina y el Caribe. La dinámica del ciclo económico actual y los desafíos de política para dinamizar la inversión y el crecimiento. Comisión Económica para América Latina y el Caribe (CEPAL). Santiago de Chile: CEPAL. Recuperado de: de: https://repositorio.cepal.org/bitstream/handle/11362/42001/159/S1700700_es.pdf

Comisión Económica para América Latina y el Caribe (CEPAL) (2018). Estudio Económico de América Latina y el Caribe, 2018. Comisión Económica para América Latina y el Caribe (CEPAL). Santiago de Chile: CEPAL

Duflo, E. \& Vinayak, A. (2012). Repensar la pobreza. Bogotá: AurusGorgi, J. (2017). Educación rezagada en América Latina pese a la alta inversión. Recuperado de: Educación rezagada en América Latina pese a alta inversión

Hanushek, E., Woessmann, L. (2007). The role of education quality for economic growth. Washington, DC: World Bank. Recuperado de: http:// documents.worldbank.org/curated/en/260461468324885735/The-role-ofeducation-quality-for-economic-growth

Heckman, J. (2004). Law and employment lessons from latinamerica and the caribbean. Washington, DC: University of Chicago Press.

Levin, H., McEwan, P., Belfield, C., Bowden A. \& Sahnd, R. (2017). Economic Evaluation in education: cost-effectiveness and benefit-cost analysis. London: SAGE

Marchesi, A. (2009). Las Metas Educativas 2021. Un proyecto iberoamericano para transformar la educación en la década de los bicentenarios. Revista Iberoamericana de Ciencia, Tecnología y Sociedad, 12 (4). 
Organización para la Cooperación y el Desarrollo Económicos (OCDE). (2008): Informe PISA 2006. Competencias cientificas para el mundo del mañana. Madrid: Santillana.

Organización de los Estados Iberoamericanos para la Educación, la Ciencia y la Cultura. (OEI). (2010). Metas educativas 2021. La educación que queremos para la generación de los bicentenarios. Madrid: OEI

Organización de las Naciones Unidas (ONU). (2011). Informe sobre Desarrollo Humano 2011. Sostenibilidad y equidad: Un mejor futuro para todos. EE.UU.: Programa Naciones Unidas para el Desarrollo (PNUD)

Organización de las Naciones Unidas (ONU). (2016). Informe sobre Desarrollo Humano 2016. Desarrollo bumano para todos. EE.UU.: Programa Naciones Unidas para el Desarrollo (PNUD)

Organización de los Estados Iberoamericanos para la Educación, la Ciencia y la Cultura. (OEI). (2016). Miradas sobre la educación en Iberoamérica. Avances y seguimiento de las Metas Educativas 2021. Madrid: OEI.

Organización de los Estados Iberoamericanos para la Educación, la Ciencia y la Cultura. (OEI). (2017). Informe Miradas 2017. Desarrollo profesional y liderazgo de directores escolares en Iberoamérica. Madrid: OEI

Organización de los Estados Iberoamericanos para la Educación, la Ciencia y la Cultura. (OEI). (2018). Miradas sobre la educación en Iberoamérica. Proyecto de revisión de los indicadores de las Metas Educativas 2021. Madrid: OEI

Sen, A. (2001). La desigualdad económica. Madrid: Fondo de cultura de España

UNESCO (2007). Educación para Todos en 2015 ¿Alcanzaremos la meta? Panorama Regional América Latina y el Caribe. París: UNESCO

UNESCO (2013). Americana Latina y Caribe. Revisión regional 2015 de la Educación para Todos. Santiago de Chile: OREALC/UNESCO

UNESCO (2015). Educación 2030. Declaración de Incheon y Marco de Acción para la realización del desarrollo sostenible 4. Garantizar una educación inclusiva y equitativa de calidad y promover oportunidades de aprendiraje permanente para todos. Foro Mundial de la Educación. Recuperado de: http://www.unesco.org/new/fileadmin/MULTIMEDIA/FIELD/Lima/pdf/INCHE_2.pd $\mathrm{f}$ 


\section{SOBRE EL AUTOR}

\section{Juan Antonio Giménez Beut}

Es Doctor en Pedagogía, Licenciado en Marketing y Diplomado en Magisterio. Tras una docencia de más de 25 años en todos los niveles educativos obligatorios se trasladó a la Universidad como profesor de los Grados de Maestro en Educación Infantil, Primaria y Pedagogía, Además imparte docencia el Máster de Formación del Profesorado en Educación Secundaria, de Salud Escolar y en Gestión y Dirección de Centros Educativos. Actualmente desempeña la función de Vicedecano del Grado de Maestro en Educación Primaria (UCV). Pertenece al "Grupo de Investigación en Pedagogía Universitaria y Estrategias de Enseñanza-Aprendizaje" (UV) que forma parte de la Red de Excelencia UNIVERSIDAD, INNOVACIÓN Y APRENDIZAJE EN LA SOCIEDAD DEL CONOCIMIENTO (2014-PN041) Ref.EDU2014-51756-REDT. Ha participado en dos proyectos competitivos de I+D+i: "EDU2012-32725" y "EDU2017-83284-R" y es miembro del comité científico del Laboratorio Montessori Aracne Editrice.

Contact information: jantonio.gimenez@ucv

Universidad Católica de Valencia "San Vicente Mártir" 